\title{
COVID-19 and Hematology—What Do We Know So Far?
}

\author{
Harshwardhan Khandait ${ }^{1}$ (D) - Garima Gandotra ${ }^{2}$ - Sonali Sachdeva ${ }^{2} \cdot$ Courtney A. Kramer $^{3} \cdot$ Derek Nye $^{3}$. \\ Reshma Golamari ${ }^{4} \cdot$ Rohit Jain ${ }^{4}$
}

Accepted: 20 October 2020 / Published online: 27 October 2020

(C) Springer Nature Switzerland AG 2020

\begin{abstract}
The severe acute respiratory syndrome-coronavirus-2 (SARS-CoV-2), the causative agent of the novel coronavirus disease 2019 (COVID 19), was reported to the World Health Organization in late 2019. This disease quickly evolved into a public health concern and was declared a pandemic on March 11,2020. COVID-19's high transmission rate and potential to cause a spectrum of systemic diseases makes it imperative for researchers and clinicians worldwide to collaborate and develop a strategy to manage and contain this disease. Studies have shown a wide range of hematological abnormalities and virus-related coagulopathies in affected patients, resulting in an increased propensity to develop serious thrombotic complications or disseminated intravascular coagulation (DIC) in severe cases. The fatal implications of coagulopathy in the form of pulmonary embolism (PE), myocardial infarction (MI), and cerebral infarction compelled us to study in-depth the pathophysiology and treatment options related to COVID-19. This analysis reviews published reports on patients with confirmed SARS-COV-2 infection and associated coagulopathy, defined as abnormalities in the coagulation parameters prothrombin time (PT), activated partial thromboplastin time (aPTT), antithrombin time, fibrinogen, fibrin degradation products, and D-dimer. In this review, we present the hematological manifestations of COVID-19, focusing on virus-associated coagulopathy and relevant pathophysiology, clinical outcomes, and treatment.
\end{abstract}

Keywords COVID-19 $\cdot$ Coagulopathy $\cdot$ SARS-CoV-2

\section{Introduction}

A series of clinically similar pneumonia cases were reported in Wuhan, China, to the World Health Organization (WHO) on December 31, 2019, by the Wuhan Municipal Health Commission. On January 9, 2020, the pathogenic cause was reported to be a novel coronavirus, and the WHO declared it a pandemic on March 11, 2021 [1]. As of September 11, 2020, the WHO has reported 27,973,127 confirmed COVID-19 cases and 905,426 deaths worldwide [1]. While COVID-19

This article is part of the Topical Collection on Covid-19

Harshwardhan Khandait

harshkhandait1@gmail.com

1 Government Medical College and Hospital, Medical Square, Nagpur 440003, India

2 Lady Hardinge Medical College, Delhi, India

3 Penn State College of Medicine, Hershey, PA, USA

4 Division of Hospital Medicine, Department of Medicine, Penn State Hershey Medical Center, Hershey, PA, USA is primarily a respiratory illness, cardiac manifestations, gastrointestinal complications, and hematological manifestations are among other features commonly reported. The most common complications reported during the course of the disease include ARDS (3.4\%), disseminated intravascular coagulation (DIC) $(0.1 \%)$ [2], cardiac injury, and multiple organ dysfunction syndrome (MODS) $[3,4]$. The most prevalent comorbidities in hospitalized patients have been found to be hypertension $(21.1 \%)$, cardiovascular diseases $(4.2 \%)$, and respiratory diseases $(2.4 \%)$, further increasing their risk of developing complications with COVID-19 [5]. This article describes hematological findings during, and complications as a result of, COVID-19 infection.

\section{Hematological Findings and Complications}

In patients with COVID-19, the most prevalent hematological findings observed in a complete blood count (CBC) include lymphopenia (83.2\%), thrombocytopenia (36.2\%), leukocytopenia (33.7\%), and neutrophilia $(34.5 \%)[2,6]$. Of note, other significant laboratory findings representing 
inflammatory markers are elevations in erythrocyte sedimentation rate (ESR) (93.8\%), serum ferritin (78.5), C-reactive protein (CRP) (60.7\%), and procalcitonin (5.5\%) [2, 6]. Median hemoglobin levels have been found to be lower in patients with severe COVID-19 disease, such as those who are admitted to an intensive care unit (ICU), who require mechanical ventilation, or who succumb to death [2].

Among the various hematological manifestations of COVID-19, coagulopathic abnormalities have recently emerged as important markers of negative prognosis, such as elevated D-dimer $(23.3 \%)$, prolonged PT $(2.1 \%)$, prolonged aPTT $(9.7 \%)$ [6]. Tang et al. demonstrated a significant difference in the levels of PT, D-dimer, and fibrin degradation products (FDP) between survivors and non-survivors of COVID-19 in patients at a Wuhan hospital [7]. Another retrospective cohort study by Zhou showed an increase in hospital mortality among patients with a D-dimer level $>1 \mu \mathrm{g} / \mathrm{dl}$ [8]. These coagulopathic factor elevations suggest a procoagulant state in COVID-19, which often manifests as both venous and arterial thrombosis, and in severe cases can progress to DIC [2]. Lupus anticoagulant has been detected with an incidence as high as $90 \%$, further supporting the presence of a hypercoagulable state [9]. Furthermore, the presence of antiphospholipid antibodies in affected patients may contribute to coagulopathy via secondary antiphospholipid syndrome [10]. A similar conclusion regarding the findings was drawn by another review by Temgoua et al. [11].

The thrombotic complications that have been observed in patients with COVID-19 include deep vein thrombosis (DVT), pulmonary embolism (PE), I.V. catheter-associated thrombosis, acute myocardial infarction (MI), limb ischemia, and cerebrovascular thrombosis $[12,13]$. In a study of critically ill patients admitted to the ICU, the cumulative incidence of thrombotic complications was reported to be $31 \%$ [14]. In patients with COVID-19, the prevalence of venous thromboembolism is approximately $20 \%$, with $3 \%$ suffering from stroke [15]. The incidence of venous thromboembolic complications, such as DVT and PE, is higher when compared to arterial thromboembolic events such as ischemic stroke, MI, or systemic arterial embolism [27\% versus $3.7 \%$, respectively] [14]. Among venous thrombosis events, the prevalence of PE has been observed to be the highest, now thought to be "in situ" thrombosis in pulmonary vessels [16]. Thus, the effect of SARS-COV2 on the hematological system is a diverse and evolving cause of concern due to its numerous complications and rising mortality.

\section{Pathophysiology}

The Coronavirus family are enveloped, single-stranded, positive-sense RNA viruses with spike glycoproteins, composed of two subunits- $\mathrm{S} 1$ and $\mathrm{S} 2$ - on their surface. The receptor-binding domain (RBD) of the SARS-CoV-2 helps to structurally differentiate it from other coronaviruses. RBD is also utilized to bind ACE- 2 receptors found in abundance on respiratory tract cells during the initial infection phase, as well as multiple other organ tissues as the disease progresses $[17,18]$. By binding to the ACE-2 receptor on Type II pneumocytes of the lung, SARS-CoV-2 can dysregulate the kallikrein/kinin system and initiate the coagulation cascade [19]. The downregulation of ACE-2 leads to angiotensin IImediated vascular dysfunction, also possibly implicated in the development of a hypercoagulable state in infected patients.

The thrombotic milieu seen with COVID-19 infection may be a result of direct endothelial and microvascular damage by the virus, followed by inflammation and the excessive release of cytokines which further aids the development of a prothrombotic state [20-22]. An increase in the complement factor $\mathrm{C} 5 \mathrm{~b}-\mathrm{C} 9$ has been shown to create extensive capillary damage in the lungs and skin of COVID-19 patients. This suggests that terminal complement activation could be the cause of endothelial damage and thrombosis in these patients [23]. Finally, an imbalance of the renin-angiotensinaldosterone system (RAAS) creates increased levels of angiotensin II, relative to angiotensin I. This imbalance manifests as unregulated inflammation and oxidative stress, leading to dysfunctional endothelium, and further contributing to thrombosis [24].

There is evidence that ACE-2 receptors are expressed on lymphocytes, which the virus utilizes to cause a direct cytotoxic effect, leading to lymphopenia [25]. COVID-19 may also attack hematopoietic stem cells in the bone marrow via the ACE-2 receptor, therefore depleting all forms of blood cells [26]. Previous studies have demonstrated that viral $\mathrm{TNF} \alpha$ induction during the cytokine storm mediates cell apoptosis [27]. Additional experiments have shown that the acidification of culture medium results in the death of a subset of T cells [28]. A similar mechanism may occur in the COVID-19 disease state, where hypoxia and blood flow stasis due to endothelial damage creates an acidic environment, resulting in immune cell apoptosis. Specific COVID-19 related morphological change has been observed in lymphocytes, with a predominance of reactive cells showing lymphoplasmacytic characteristics [29].

Zini et al. observed changes in circulating neutrophils and platelets, suggesting the existence of disturbed myelopoiesis in patients with severe, systemic COVID-19 infection [30]. A review by Violetis concluded that the neutrophilia could be due to the "cytokine storm." It could also be an indicator of a superimposed bacterial infection [31]. In addition to platelet structural changes, a reduction in absolute platelet count has been observed. These findings could be attributed to COVID19 infection destroying hematopoietic precursors in the marrow and megakaryocytes in the lungs. Another proposed 
mechanism is the destruction of platelets by virus-induced autoantibodies, which form immune complexes, and are then cleared from the body. In addition to these potential processes, the consumption of platelets during the coagulation cascade and thrombi formation further lowers platelet levels [32].

The clinical manifestations and laboratory findings of COVID-19 are similar to those seen in secondary hemophagocytic lymphohistiocytosis (sHLH), which is defined by $\mathrm{T}$ cell overactivation leading to the production of granulocyte-macrophage colony-stimulating factor (GM-CSF) and interleukin-6 (IL-6). GM-CSF stimulates CD14+ CD16+ inflammatory mononuclear macrophages to produce IL-6 and a plethora of other inflammatory mediators [33]. This phenomenon may be responsible for the mass destruction of immune cells via activation of mononuclear macrophage system and excessive cytokine release. Figure 1 depicts the pathophysiology of hematologic changes in COVID-19.

\section{Clinical Outcomes}

Hematological laboratory findings can be utilized to determine the severity and prognosis of COVID-19 infection. Thrombocytopenia has been shown to be associated with an increased risk of severe disease and COVID-19-related mortality [34]. In a meta-analysis by Zhao et al., lymphopenia was linked to a threefold increase of severe COVID-19 disease [35]. Wu et al. assessed risk factors associated with ARDS and death in patients with COVID-19 and reported that an increase in neutrophils was directly linked to adverse outcomes and mortality [6].

Abnormalities in coagulation parameters, such as increased prothrombin time (PT) and activated partial thromboplastin time (aPTT), elevated fibrinogen, fibrin degradation products (FDP), and D-dimer levels, have also been shown to be important prognostic factors in patients with COVID-19 pneumonia [7]. Numerous studies focusing on hematologic

\section{Pathophysiology of Hematologic Changes in COVID-19}
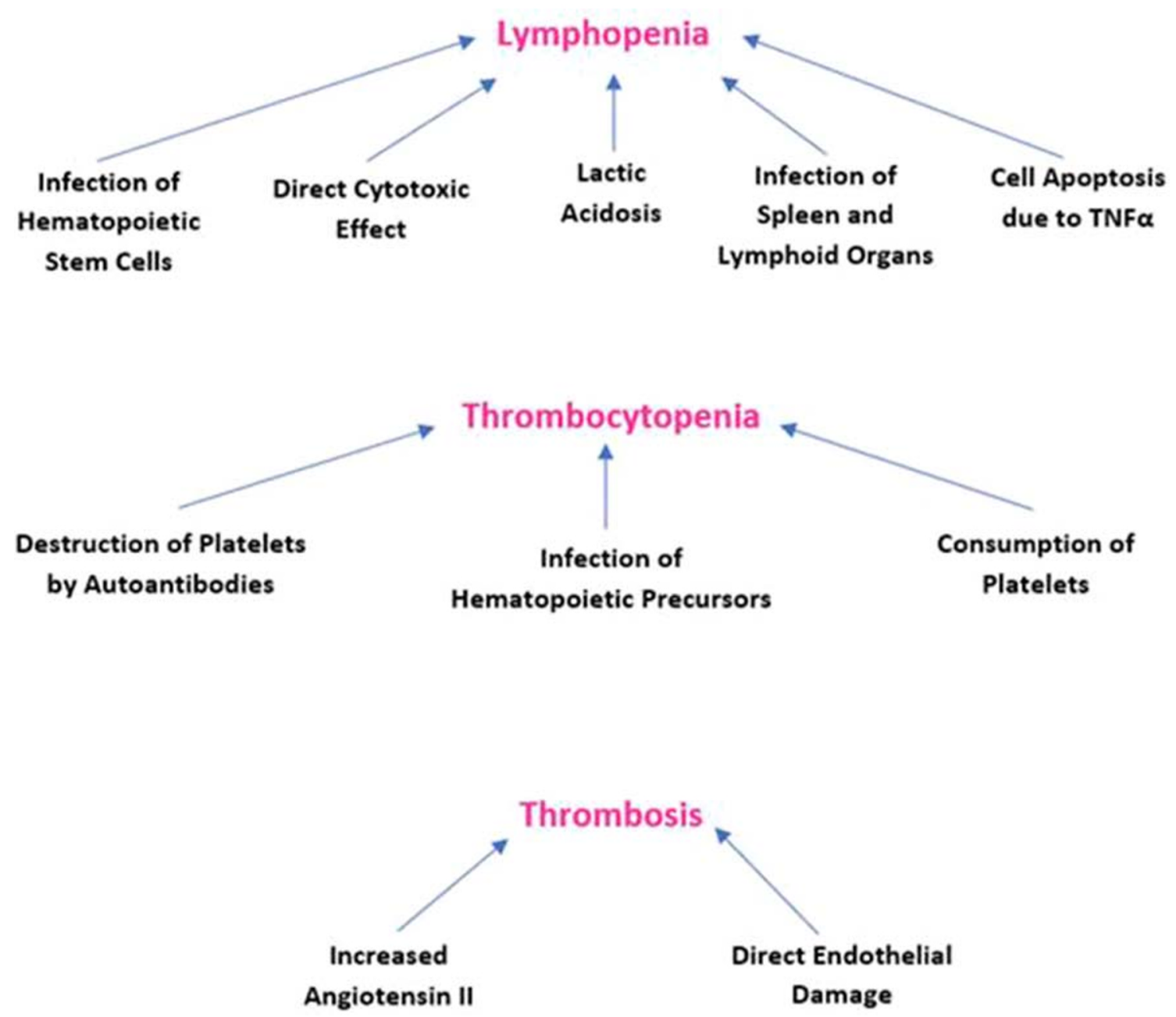

Fig. 1 Pathophysiology of hematologic changes in COVID-19 
abnormalities in COVID-19 patients indicate the presence of a co-existing coagulopathy, which may predispose to thrombotic complications, including venous thromboembolism (VTE), $\mathrm{PE}$, arterial thromboembolism, MI, cerebral infarction, and DIC [36]. There has also been documentation of in situ thrombus formation in the coronary circulation, leading to MI [37]. Bangalore et al. reported 18 cases of COVID-19 with STelevation MI and elevated D-dimer levels, suggesting the role of COVID-19-associated coagulopathy in coronary thrombosis [38].

There is also an emerging role for biomarkers, such as LDH, procalcitonin, ferritin, IL-6, and cardiac troponin-1, which have been found to be significantly elevated in nonsurvivors compared to survivors of COVID-19 [8]. Plasma levels of CRP have also been shown to correlate with disease lethality and indicate poor survival chances in COVID-19 [39]. A case series by Liu et al. demonstrated that increasing eosinophils may be an indicator of improvement in the COVID-19 disease [40]. It has also been noted that hypoalbuminemia is a predictor of mortality, independent of age and comorbidity burden [41]. Furthermore, a rise in neutrophil/ lymphocyte count and neutrophil/platelet count may point towards a heightened risk of myocardial injury and mortality [42].

\section{Treatment}

The American Society of Hematology has recommended the use of either low molecular weight heparin (LMWH) or fondaparinux for thromboprophylaxis in COVID-19associated hypercoagulability, except in cases where the risk of bleeding supersedes thrombosis risk [38]. In those with existing contraindications for anticoagulation, pneumatic compression devices could be initiated instead. A regulatory agency approved regimen may be used for thromboprophylaxis after discharge, such as a first dose of betrixaban $160 \mathrm{mg}$, followed by $80 \mathrm{mg}$ daily for 35-42 days, or rivaroxaban $10 \mathrm{mg}$ daily for 31-39 days [38]. Therapeutic anticoagulation is initiated in patients with confirmed cases of VTE, with patient comorbidities and co-existing conditions dictating the choice of treatment- either low molecular weight heparin, unfractionated heparin, or direct anticoagulants. If necessary, reduced antithrombin III levels can be replenished with fresh frozen plasma [43].

PE management in COVID-19 patients follows a standardized guideline with hemodynamically stable patients receiving anticoagulation with close monitoring and severe cases receiving fibrinolysis. In unstable patients or if systemic fibrinolysis is contraindicated, catheter-directed therapies can be utilized. Patients with COVID-19-associated coagulopathy should be evaluated with viscoelastic coagulation tests including thromboelastography (TEG) and a coagulation and platelet function analyzer [43]. Patients with prolonged PT or APTT $>1.5$ times, TEG $\mathrm{R}$ time $>10 \mathrm{~min}$, are candidates for fresh frozen plasma infusion [43].

In acute coronary syndrome with plaque rupture, the use of dual antiplatelet and anticoagulants is recommended in accordance with standard guidelines, unless contraindicated [44]. Differentiation between myocarditis, nonspecific myocardial injury, and plaque rupture is important as the former two conditions do not require intervention [45]. Transthoracic echocardiography (TTE) can be utilized prior to intervention to assess regional wall motion abnormalities. In ST-elevation myocardial infarction (STEMI), the risk (of transmission and delay in treatment) benefit ratio must be considered and selected cases may receive fibrinolysis [45]. In STEMI, the decision to proceed to the catheterization laboratory is guided by the severity of STEMI, the severity of COVID-19 in patients, and the risk of transmission.

Heparin is generally avoided in DIC but is recommended in DIC associated with COVID-19 [14]. Unless clinically necessary, long-acting antiplatelet drugs should be discontinued [36]. In cases with active bleeding, the transfusion of blood products can be considered, with platelet concentrate administered to maintain counts $>50 * 109 / 1$ and fresh frozen plasma in patients with deranged PT/ APTT ratio or decreased fibrinogen [36].

The pathophysiology of thrombosis in COVID-19 is unique; therefore, its management through standardized therapy cannot be validated. A study by Klok et al. failed to demonstrate the benefit of prophylactic LMWH in critically ill patients admitted to the ICU [14]. Therefore, it cannot be assumed that prophylactic LMWH in standard doses is the standard of care in preventing thrombotic complications associated with COVID-19. We suggest the use of VTE risk stratification systems for COVID-19 patients, such as the Caprini score and Padua Model [45]. The British Society of Hematology recommends using the ISTH DIC score for prognosticating patients with severe COVID-19 infection and tailoring the treatment accordingly [46]. Large-scale, randomized trials will be required to establish treatment guidelines and also determine whether anti-inflammatory drugs may be beneficial, considering that inflammation plays an important role in COVID-19-associated thrombosis.

\section{Conclusion}

Initially thought to affect predominantly the lungs, COVID-19 is a systemic disease with the potential to affect numerous organs systems. The role of laboratory parameters is increasingly being utilized in the diagnosis, prediction of adverse clinical outcomes, and prognosis in hospitalized COVID-19 patients. Given the strong prognostic value of hematologic abnormalities and their relative ease in monitoring, they 
should be meticulously followed in all patients and can help in providing urgent intensive care referral for those who are at a greater risk. COVID-19-associated coagulopathy is an important predictor of mortality and can lead to VTE, arterial thromboembolism, MI, cerebral infarction, and DIC. Furthermore, due to the intensive involvement of COVID-19 in the coagulation system, it can be a target to develop future therapies against COVID-19. Knowledge about COVID-19 is still rapidly evolving and large-scale clinical trials are warranted to assess the effect of SARS-COV 2 on the hematological system and guide the development of treatment options.

Author Contributions Study concept and design: Rohit Jain, Reshma Golamari

Literature search: Harshwardhan Khandait, Garima Gandotra, Sonali Sachdeva, Courtney Kramer

Drafting of the manuscript: Harshwardhan Khandait, Garima Gandotra, Sonali Sachdeva, Courtney Kramer, Derek Nye

Critical revision of the manuscript for important intellectual content: Harshwardhan Khandait, Garima Gandotra, Sonali Sachdeva, Rohit Jain, Reshma Golamari

\section{Compliance with Ethical Standards}

Conflict of Interest The authors declare that they have no conflict of interests.

Informed Consent The data used in this study are publicly available and de-identified database thus informed consent or IRB approval was not needed for this study.

\section{References}

1. Timeline of WHO's response to COVID-19. World Health Organization, World Health Organization, www.who.int/newsroom/detail/29-06-2020-covidtimeline. Accessed 29 June 2020.

2. Guan WJ, Ni ZY, Hu Y, Liang WH, Ou CQ, He JX, et al. Clinical characteristics of coronavirus disease 2019 in China. N Engl J Med. 2020;382(18):1708-20.

3. Harapan H, Itoh N, Yufika A, Winardi W, Keam S, Te H, et al. Coronavirus disease 2019 (COVID-19): a literature review. J Infect Public Health. 2020;13(5):667-73.

4. Gerges Harb J, Noureldine HA, Chedid G, Eldine MN, Abdallah DA, Chedid NF, et al. SARS, MERS and COVID-19: clinical manifestations and organ-system complications: a mini review. Pathog Dis. 2020;78(4). https://doi.org/10.1093/femspd/ftaa033.

5. Yang J, Zheng Y, Gou X, Pu K, Chen Z, Guo Q, et al. Prevalence of comorbidities and its effects in patients infected with SARS-CoV-2: a systematic review and meta-analysis. Int J Infect Dis. 2020;94: 91-5.

6. Wu C, Chen X, Cai Y, Xia J, Zhou X, Xu S, et al. Risk factors associated with acute respiratory distress syndrome and death in patients with coronavirus disease 2019 pneumonia in Wuhan. China JAMA Intern Med. 2020;180(7):1-11.

7. Tang N, Li D, Wang X, Sun Z. Abnormal coagulation parameters are associated with poor prognosis in patients with novel coronavirus pneumonia. J Thromb Haemost. 2020;18(4):844-7.

8. Zhou F, Yu T, Du R, Fan G, Liu Y, Liu Z, et al. Clinical course and risk factors for mortality of adult inpatients with COVID-19 in
Wuhan, China: a retrospective cohort study. Lancet. 2020;395(10229):1054-62.

9. Helms J, Tacquard C, Severac F, Leonard-Lorant I, Ohana M, Delabranche X, et al. High risk of thrombosis in patients with severe SARS-CoV-2 infection: a multicenter prospective cohort study. Intensive Care Med. 2020;46(6):1089-98.

10. Cavalli E, Bramanti A, Ciurleo R, Tchorbanov AI, Giordano A, Fagone P, et al. Entangling COVID-19 associated thrombosis into a secondary antiphospholipid antibody syndrome: diagnostic and therapeutic perspectives (review). Int J Mol Med. 2020;46(3):90312 .

11. Temgoua MN, Endomba FT, Nkeck JR, Kenfack GU, Tochie JN, Essouma M. Coronavirus disease 2019 (COVID-19) as a multisystemic disease and its impact in low- and middle-income countries (LMICs). SN Compr Clin Med. 2020;2:1377-87.

12. Gupta A, Madhavan MV, Sehgal K, Nair N, Mahajan S, Sehrawat TS, et al. Extrapulmonary manifestations of COVID-19. Nat Med. 2020;26(7):1017-32.

13. Shi W, Lv J, Lin L. Coagulopathy in COVID-19: focus on vascular thrombotic events. J Mol Cell Cardiol. 2020;146:32-40.

14. Klok FA, Kruip M, van der Meer NJM, Arbous MS, Gommers D, Kant KM, et al. Incidence of thrombotic complications in critically ill ICU patients with COVID-19. Thromb Res. 2020;191:145-7.

15. Al-Ani F, Chehade S, Lazo-Langner A. Thrombosis risk associated with COVID-19 infection. A scoping review. Thromb Res. 2020;192:152-60.

16. Gabrielli M, Lamendola P, Esperide A, Valletta F, Franceschi F. COVID-19 and thrombotic complications: pulmonary thrombosis rather than embolism? Thromb Res. 2020;193:98.

17. Ragab D, Salah Eldin H, Taeimah M, Khattab R, Salem R. The COVID-19 cytokine storm: what we know so far. Front Immunol. 2020;11:1446.

18. Weiss SR, Navas-Martin S. Coronavirus pathogenesis and the emerging pathogen severe acute respiratory syndrome coronavirus. Microbiol Mol Biol Rev. 2005;69(4):635-64.

19. van Nieuwkoop C. COVID-19 associated pulmonary thrombosis. Thromb Res. 2020;191:151.

20. Ackermann M, Verleden SE, Kuehnel M, Haverich A, Welte T, Laenger F, et al. Pulmonary vascular endothelialitis, thrombosis, and angiogenesis in Covid-19. N Engl J Med. 2020;383(2):120-8.

21. Varga Z, Flammer AJ, Steiger P, Haberecker M, Andermatt R, Zinkernagel AS, et al. Endothelial cell infection and endothelitis in COVID-19. Lancet. 2020;395(10234):1417-8.

22. Du F, Liu B, Zhang S. COVID-19: the role of excessive cytokine release and potential ACE2 down-regulation in promoting hypercoagulable state associated with severe illness. J Thromb Thrombolysis. 2020:1-17. https://doi.org/10.1007/s11239-02002224-2.

23. Magro C, Mulvey JJ, Berlin D, Nuovo G, Salvatore S, Harp J, et al. Complement associated microvascular injury and thrombosis in the pathogenesis of severe COVID-19 infection: a report of five cases. Transl Res. 2020;220:1-13.

24. Tikellis C, Thomas MC. Angiotensin-converting enzyme 2 (ACE2) is a key modulator of the renin angiotensin system in health and disease. Int J Pept. 2012;2012:256294.

25. Xu H, Zhong L, Deng J, Peng J, Dan H, Zeng X, et al. High expression of ACE2 receptor of 2019-nCoV on the epithelial cells of oral mucosa. Int J Oral Sci. 2020;12(1):8.

26. Jokubaitis VJ, Sinka L, Driessen R, Whitty G, Haylock DN, Bertoncello I, et al. Angiotensin-converting enzyme (CD143) marks hematopoietic stem cells in human embryonic, fetal, and adult hematopoietic tissues. Blood. 2008;111(8):4055-63.

27. Liao Y-C, Liang W-G, Chen F-W, Hsu J-H, Yang J-J, Chang M-S. IL-19 induces production of IL- 6 and TNF- $\alpha$ and results in cell apoptosis through TNF- $\alpha$. J Immunol. 2002;169(8):4288-97. 
28. Fischer K, Hoffmann P, Voelkl S, Meidenbauer N, Ammer J, Edinger M, et al. Inhibitory effect of tumor cell-derived lactic acid on human T cells. Blood. 2007;109(9):3812-9.

29. Fan BE, Chong VCL, Chan SSW, Lim GH, Lim KGE, Tan GB, et al. Hematologic parameters in patients with COVID-19 infection. Am J Hematol. 2020;95(6):E131-E4.

30. Zini G, Bellesi S, Ramundo F, d'Onofrio G. Morphological anomalies of circulating blood cells in COVID-19. Am J Hematol. 2020;95(7):870-2.

31. Violetis OA, Chasouraki AM, Giannou AM, et al. COVID-19 infection and haematological involvement: a review of epidemiology, pathophysiology and prognosis of full blood count findings. SN Compr Clin Med. 2020;2:1089-93.

32. Zhou Y, Fu B, Zheng X, Wang D, Zhao C, Qi Y, et al. Pathogenic $\mathrm{T}$-cells and inflammatory monocytes incite inflammatory storms in severe COVID-19 patients. Natl Sci Rev. 2020;7(6):998-1002. https://doi.org/10.1093/nsr/nwaa041.

33. Zhang Y, Zeng X, Jiao Y, Li Z, Liu Q, Ye J, Yang M. Mechanisms involved in the development of thrombocytopenia in patients with COVID-19. Thromb Res. 2020;193:110-5. https://doi.org/10. 1016/j.thromres.2020.06.008.

34. Lippi G, Plebani M, Henry BM. Thrombocytopenia is associated with severe coronavirus disease 2019 (COVID-19) infections: a meta-analysis. Clin Chim Acta. 2020;506:145-8.

35. Zhao Q, Meng M, Kumar R, Wu Y, Huang J, Deng Y, et al. Lymphopenia is associated with severe coronavirus disease 2019 (COVID-19) infections: a systemic review and meta-analysis. Int J Infect Dis. 2020;96:131-5.

36. Bikdeli B, Madhavan MV, Jimenez D, Chuich T, Dreyfus I, Driggin E, et al. COVID-19 and thrombotic or thromboembolic disease: implications for prevention, antithrombotic therapy, and follow-up: JACC state-of-the-art review. J Am Coll Cardiol. 2020;75(23):2950-73.

37. Dominguez-Erquicia P, Dobarro D, Raposeiras-Roubín S, BastosFernandez $\mathrm{G}$, Iñiguez-Romo A. Multivessel coronary thrombosis in a patient with COVID-19 pneumonia. Eur Heart J. 2020;41(22): 2132.
38. COVID-19 and VTE/anticoagulation: frequently asked questions. COVID-19 and VTE-anticoagulation - Hematology.org. https:// www.hematology.org/covid-19/covid-19-and-vte-anticoagulation. Accessed August 5, 2020.

39. Sahu BR, Kampa RK, Padhi A, Panda AK. C-reactive protein: a promising biomarker for poor prognosis in COVID-19 infection. Clin Chim Acta. 2020;509:91-4.

40. Liu F, Xu A, Zhang Y, Xuan W, Yan T, Pan K, et al. Patients of COVID-19 may benefit from sustained Lopinavir-combined regimen and the increase of eosinophil may predict the outcome of COVID-19 progression. Int J Infect Dis. 2020;95:183-91.

41. Li J, Li M, Zheng S, Li M, Zhang M, Sun M, et al. Plasma albumin levels predict risk for nonsurvivors in critically ill patients with COVID-19. Biomark Med. 2020;14:827-37.

42. Guo T, Fan Y, Chen M, Wu X, Zhang L, He T, et al. Cardiovascular implications of fatal outcomes of patients with coronavirus disease 2019 (COVID-19). JAMA Cardiol. 2020;5(7):1-8.

43. Song JC, Wang G, Zhang W, Zhang Y, Li WQ, Zhou Z. Chinese expert consensus on diagnosis and treatment of coagulation dysfunction in COVID-19. Mil Med Res. 2020;7(1):19.

44. Amsterdam EA, Wenger NK, Brindis RG, Casey DE Jr, Ganiats TG, Holmes DR Jr, et al. 2014 AHA/ACC guideline for the management of patients with non-ST-elevation acute coronary syndromes: a report of the American College of Cardiology/ American Heart Association Task Force on Practice Guidelines. J Am Coll Cardiol. 2014;64(24):e139-228.

45. Liu X, Liu C, Chen X, Wu W, Lu G. Comparison between Caprini and Padua risk assessment models for hospitalized medical patients at risk for venous thromboembolism: a retrospective study. Interact Cardiovasc Thorac Surg. 2016;23(4):538-43.

46. Kinasewitz GT, Zein JG, Lee GL, Nazir SA, Taylor FBJ. Prognostic value of a simple evolving disseminated intravascular coagulation score in patients with severe sepsis*. Crit Care Med. 2005;33(10):2214-21.

Publisher's Note Springer Nature remains neutral with regard to jurisdictional claims in published maps and institutional affiliations. 